\title{
EXPRESSION OF TLR7 IN ORAL SQUAMOUS CELL CARCINOMA AND ITS CORRELATION WITH LYMPH NODE METASTASIS (AN IMMUNO- HISTOCHEMICAL STUDY)
}

\author{
Engy A. Youssef ${ }^{1} M S c$, Hanaa S. Raslan ${ }^{2} P h D$, Sahar M. El-Sheikh ${ }^{2} P h D$, Sahar E. Riad ${ }^{2} P h D$, \\ Radwa A. Mehanna ${ }^{3} P h D$ Marwa M. Afifi ${ }^{4} P h D$
}

\begin{abstract} prognosis and aid in treatment modalities. correlate it with the lymph node involvement. complex method (LSAB), using the anti-TLR7 antibody. metastasis. KEYWORDS: Oral squamous cell carcinoma, lymph node metastasis, TLR7, Immunohistochemistry.

1. Assistant lecturer in Oral Pathology Department, Faculty of Dentistry, Kafrelsheikh University.

2. Professor in Oral Pathology Department, Faculty of Dentistry, Alexandria University.

3. Assistant Professor in Physiology Department, Faculty of Medicine, Alexandria University.

4. Lecturer in Oral Pathology Department, Faculty of Dentistry, Alexandria University.
\end{abstract}

INTRODUCTION: Oral squamous cell carcinoma (OSCC) accounts for most of oral cancers which is about (90\%) of all types. OSCC is an important cause of morbidity and mortality worldwide. Early detection; diagnosis and early treatment are still the key points for OSCC patients to improve survival rate and quality of life. Toll like receptors (TLRs), through their role in innate immunity, have been implicated in the pathogenesis of several diseases, including cancer and OSCC is one of these cancers. Nodal metastasis is playing a key role in detecting the prognosis and the outcome. TLR7 is one of the TLRS which is expressed in OSCC, the correlation between the expression of this receptor and the presence of nodal metastasis has been assessed and evaluated as TLR7 may be of great significance in tumors metastasis so can help in

OBJECTIVES: The aim of the present study was to evaluate the expression of TLR7 in the specimens of primary tumor tissues of OSCC and

MATERIALS AND METHODS: TLR7expression was studied in 50 OSCC cases. The specimens were taken from the primary tumor of 25 cases with positively involved lymph nodes and another 25 cases with negatively involved lymph nodes and 10 normal mucosal tissues taken from OSCC patients and healthy individuals. Immuno-histochemical (IHC) staining was performed using the Labeled Strept-Avidin Biotin

RESULTS: TLR7 showed high expression in OSCC cases with positive lymph node metastasis than those with negative lymph node

CONCLUSIONS: TLR7 expression could be a guiding tool used to detect prognosis in OSCC patients and as an indicator for metastasis.

Corresponding author:

E-mail: injymourad @gmail.com

\section{INTRODUCTION}

Oral cancer, a subtype of head and neck cancer (1). It is a cancerous tissue growth located in the oral cavity (2). It may arise as a primary lesion originating from the oral tissues or by metastasis from a distant site of origin, or by extension from a neighboring anatomic structure, such as the nasal cavity (3). Approximately 30\% of head and neck cancers are oral cavity cancers, with nearly $95 \%$ of the latter being oral squamous cell carcinomas (OSCC) (4). Oral squamous cell carcinoma (OSCC) is the sixth most prevalent malignancy worldwide (5). The prognosis of OSCC remains dismal because more than $50 \%$ of patients die from this disease or its complications within 5 years with current therapies (6). Lymph node (LN) metastasis is one of the most implicated and effective factors in the treatment and prognosis of patients with OSCC as LN metastasis are a strong determinant for a poor outcome and bad prognosis. It reduces the overall 5 years survival by nearly half $(7,8)$.

Recently, there has been a growing recognition of interest in anti-tumor functions initiated by the innate immune response. The role of Toll-like receptors (TLRs) is among the frontiers of exploration (9). Toll like receptors (TLRs) are a family of pattern recognition receptors (PRRs) that function as primary sensors of the innate immune system to recognize microbial pathogens. Toll-like receptors (TLRs) were first discovered in drosophila, in the membranes of binding PRRs and are known to target a series of mechanisms leading to the synthesis and secretion of cytokines and activation of other host defense programs that are crucial to the development of innate or adaptive immunity (10). Ten functional TLRs have been identified in humans (TLR1-10) (12). These receptors are highly expressed on the cells of the immune system as well as nonimmune cells such as the epithelial cells in respiratory, genitourinary and gastrointestinal epithelium and skin as these areas are considered as the first line of defense also endothelial cells and fibroblasts. $(11,12,13,14)$.

TLR7, a receptor that recognizes viral single-stranded RNA, is located in the endosomal compartments of plasmacytoid dendritic cells (pDCs), B lymphocytes, natural killer cells, and virally infected cells (15). It has also been reported to be expressed by a variety of cancer cells. TLR7 shows a wide behavior in the tumor microenvironment with either pro-tumorigenic or antitumorigenic effects. However, the distinct expression and 
function of TLR7 in head and neck tumors, especially OSCC, are unclear (16).

To the best of our knowledge, no researches in literature are available for the association of TLR7 expression in OSCC and metastasis potentiality. This study was thus conducted to correlate the immune-histochemical expression of TLR7 in OSCC cases to LN metastasis and so such a protein marker may aid in treatment modalities.

\section{MATERIALS AND METHODS}

\section{Study material}

The present study was performed in the Oral Pathology Department, Faculty of Dentistry, Alexandria University after gaining the approval of the Research Ethics Committee. Fifty OSCC cases collected from the CranioMaxillofacial and Plastic Surgery Department were included. Biopsies were taken from the primary tumor of twenty-five cases proved to have positive lymph nodes and another twenty- five cases with negative lymph nodes. In addition, 10 specimens were taken from the healthy individuals with normal oral mucosa who were indicated for alveoloplasty served as a control group. The biopsies were confirmed histo-pathologically in the Oral Pathology Department at the Faculty of Dentistry, Alexandria University. The biopsies of the patients and bio-archiving were in compliance with the Code of Professional Ethics for Dentistry adopted by the Alexandria University, Faculty of Dentistry.

\section{Histopathological and immuno-histochemical analysis}

The specimens were fixed in $10 \%$ neutral buffered formalin, processed and embedded in paraffin wax using the conventional procedures. Serial sections of 3-4 $\mu \mathrm{m}$ thickness were placed on glass slides and stained using Hematoxylin and Eosin (H\&E). Immunohistochemical (IHC) staining using the anti-TLR7 antibody (the primary polyclonal rabbit anti-human TLR 7 antibody (ab45371; Abcam, USA) was performed using the Labeled StreptAvidin Biotin complex method (LSAB) (17). The sections were then examined and the intensity of the immunostaining was quantified in terms of both the mean area percent (AP) and the mean optical density (OD) by the computer image analyzer ImageJ software (NIH, USA).

\section{Statistical analysis of the Data}

Statistical package for social sciences version 20.0 (SPSS 20.0, SPSS Inc., Chicago, IL, USA) was carried out for the statistical analyses and calculations of data (18). The differences in the mean OD and AP in OSCC cases with positive and negative lymph nodes were estimated and calculated using the student (t) test. Any P-values equal to or less than 0.05 were considered statistically significant. All the data are expressed as mean \pm SD (standard deviation).

\section{RESULTS}

\section{Clinical Results}

The demographic data of the patients included in the current study showed that: The age of the patients ranged from (44$\mathbf{8 1}$ years). The mean age was found to be (61 years) while the median age was (59.5 years). Thirty-two patients (64\%) were females and eighteen (36\%) were males.

The most common site of occurrence was the lateral side of the tongue $(\mathbf{5 2 \%}, \mathbf{n = 2 6})$. This is followed by the buccal mucosa representing $(\mathbf{1 8 \%}, \mathbf{n}=\mathbf{9})$ and the alveolar mucosa $(\mathbf{1 2 \%}, \mathbf{n}=\mathbf{6})$. Other sites included in the present work were the retromolar area $(\mathbf{6 \%}, \mathbf{n = 3})$, the palate $(\mathbf{6 \%}$, $\mathbf{n = 3}$ ) and the floor of the mouth $(6 \%, n=3)$.

\section{Histopathological Results}

The microscopical examination of OSCC revealed that $(\mathbf{2 2 \%}, \mathbf{n}=\mathbf{1 1})$ were of the well differentiated type, $(\mathbf{6 4 \%}$, $\mathbf{n = 3 2}$ ) were moderately differentiated, $(\mathbf{1 4 \%}, \mathbf{n}=\mathbf{7})$ were of the poorly differentiated type.

\section{Immuno-histochemical Results}

All biopsies of normal oral mucosa $(n=10)$ showed weak immunoreactivity for TLR7 which was limited to the basal and the parabasal layers. (Figure 1)

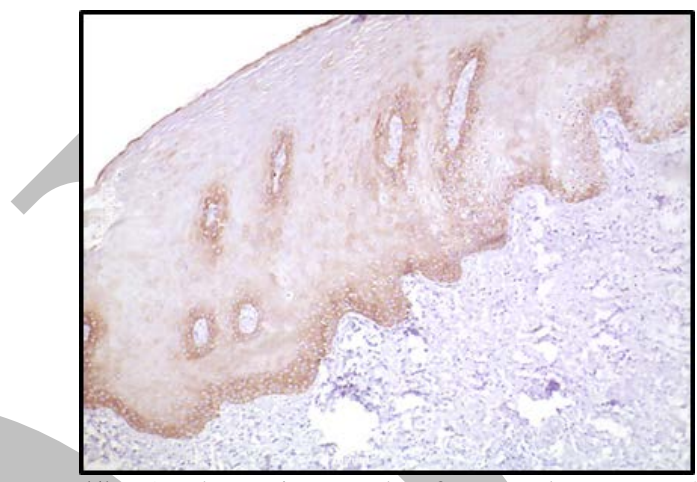

Figure (1): A Photomicrograph of Normal Mucosa Showing Weak TLR7 Immuno-signals Confined to the Basal and Para-basal Layers. (X 200)

All the tumor biopsies showed immunoreactivity to the anti-TLR7 and it was in the cytoplasm of the tumor cells as well as the nuclei with varying intensities. The expression with varying intensities ranging from weak to moderate reactions in the $\mathrm{LN}$ negative cases and moderate to severe reactions in the $\mathrm{LN}$ positive cases. (Figures 2-4)

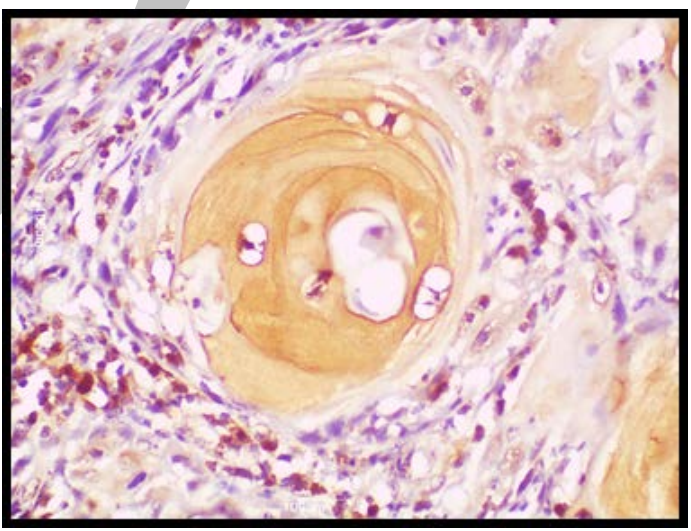

Figure (2): A Photomicrograph of a Well Differentiated OSCC Case with Negative LN Status Showing Weak TLR7Immunosignals. (X400) 


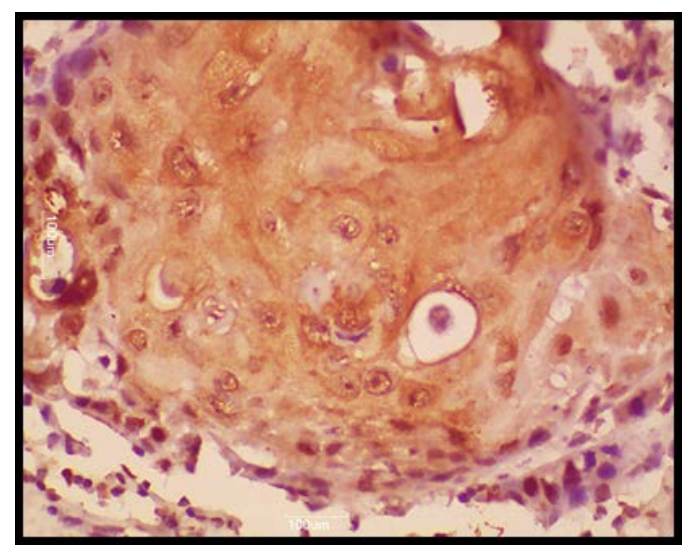

Figure (3): A Photomicrograph of a Moderately Differentiated OSCC Case with Positive LN status Showing positively Intense Cytoplasmic and nuclear TLR7 Immuno-signals. (x400)

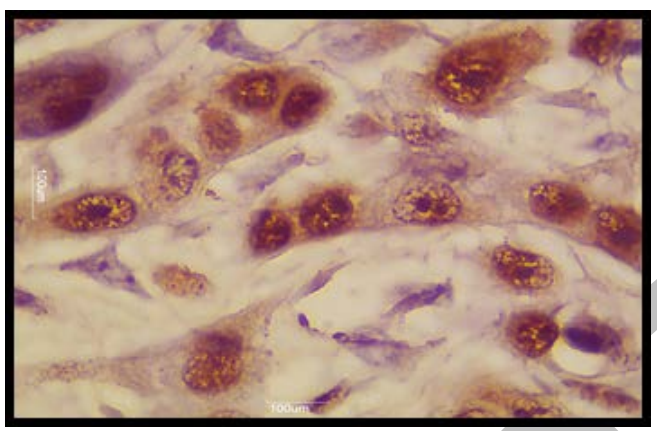

Figure (4): A Photomicrograph of a Poorly Differentiated OSCC with Positive LN status Showing Positively intense Cytoplasmic and nuclear TLR7 Immuno-signals in the malignant pleomorphic Cells. (X1000)

\section{Correlation of TLR7 expression with the lymph node involvement in OSCC}

OSCC cases with positive LNs showed higher TLR7 immunoreactivity than the negative LN cases. The expression of TLR7 immunostaining was calculated in terms of mean area percent (AP) and mean optical density (OD). The mean AP was $(88.06 \pm 3.39)$ for the $L N$ positive group and (49.86 \pm 13.62$)$ for the $\mathrm{LN}$ negative group. The mean OD was also higher in the LN positive group (56.56 \pm 4.89) than the LN negative group (41.63 \pm 8.05$)$. The difference between these two groups regarding both AP and OD revealed statistical significance at $(\mathrm{P} \leq 0.05)$ using the student t-test. (Tables 1\&2)

Table (1): Comparison between Area Percent of TLR7 Immunostaining in Negative and Positive LN Metastasis

\begin{tabular}{|c|c|c|c|c||}
\hline Mean AP & $\begin{array}{c}\text { LN -ve } \\
(\mathbf{n = 2 5 )}\end{array}$ & $\begin{array}{c}\text { LN +ve } \\
(\mathbf{n}=\mathbf{2 5})\end{array}$ & $\mathbf{t}$ & $\mathbf{P}$ \\
\cline { 1 - 3 } Min. - Max. & $31.15-71.41$ & $82.56-92.17$ & \multirow{2}{*}{7.7421} & $<0.0001^{*}$ \\
\cline { 1 - 3 } Mean & 49.86 & 88.06 & & \\
\hline SD & 13.62 & 3.39 & & \\
\hline
\end{tabular}

$\mathrm{t}, \mathrm{p}$ : $\mathrm{t}$ and $\mathrm{p}$ values for Student t-test for comparing between the two groups

*: Statistically significant at $\mathrm{p} \leq 0.05$
Table (2): Comparison between Optical Density of TLR7 Immunostaining in Negative and Positive LN Metastasis

\begin{tabular}{|c|c|c|c|c|}
\hline $\begin{array}{c}\text { Mean } \\
\text { OD }\end{array}$ & $\begin{array}{c}\text { LN -ve } \\
\text { (n=25) }\end{array}$ & $\begin{array}{c}\text { LN +ve } \\
\text { (n=25) }\end{array}$ & t & p \\
\hline Min. - & $28.96-$ & $\begin{array}{c}48.62- \\
7.0895\end{array}$ & $<0.0001^{*}$ \\
Max. & 58.39 & 62.51 & & \\
\hline Mean & 41.63 & 56.56 & & \\
\hline SD & 8.05 & 4.89 & & \\
\hline
\end{tabular}

$\mathrm{t}, \mathrm{p}$ : $\mathrm{t}$ and $\mathrm{p}$ values for Student t-test for comparing between the two groups

*: Statistically significant at $\mathrm{p} \leq 0.05$

\section{DISCUSSION}

Up till now, early detection, diagnosis and early treatment are still the key points for OSCC patients to improve survival rate and quality of life. Besides routine clinical examination, laboratory and image examinations are considered to be useful for early detection and early diagnosis (19). With advances in molecular biology and immunology, researches on tumor markers in tissues have been gradually deepened. Their detection and correlation with OSCC have been suggested to be potentially useful for clinical diagnosis and prognostic monitoring. Tumorderived markers are genes or proteins that play a direct role in carcinogenesis, and may sometimes also be used as biomarkers to improve cancer detection, predict disease outcome or response to therapy $(20,21)$.

TLRs function as double-edged swords, with both pro and anti-tumor consequences. However, the exact mechanisms by which TLRs interact with tumor cells and how these cells are able to escape immunological eradication have only recently started to unravel. Therefore, understanding the roles of TLRs in tumor biology may pave the way for the discovery of novel therapeutic targets in cancer therapy (22-24). TLR7 has a role in innate immune response and at the same time contribute to tumor progression.

To the best of our knowledge and after expanded search and exploration in the literature, no studies were available for the association of TLR7 expression in OSCC and metastasis potentiality therefore, this study was conducted to correlate the immune-histochemical expression of TLR7 in OSCC cases to LN metastasis in Egyptian population and so such a protein marker may aid in treatment modalities.

In the current study, the immuno-histochemical expression of TLR7 protein was significantly higher in the cases that were associated with metastatic LNs compared to those without LN metastasis. This suggests an association between the expression of TLR7 in the tumor cells and their ability for invasion and metastasis. In contrast to these findings, Farzaneh et al. (25) and Shiva et al. (26) postulated in their studies that significant increase in TLR2, TLR4, TLR7 in OSCC samples in the tongue was (70.1\%, 72.4\%, $66.7 \%$ ) respectively and tumors with high TLR2 and TLR4 expression only were significantly associated with a higher probability of lymph node metastasis and increased depth of invasion also. In accordance to these results also Ilyar et al. (27) evaluated the expression of TLR 3, 4, 7, 9 in esophageal squamous cell carcinoma and he found that TLR4 also is the only one associated with a higher probability of lymph node metastasis and increased depth of invasion.

Julien et al. (28) stated that stimulation and triggering of TLR7 expressed by human lung cancer cells induces cell 
survival and chemo-resistance. In agreement to this finding Saradiya et al. (29) confirmed the fact that TLR7 promotes tumor progression, chemotherapy resistance, and poor clinical outcomes in non-small cell lung cancer.

TLR7 agonists are under investigation for their ability to enhance antitumor immune responses. Mee et al. (30) investigated that imiquimod, TLR7 agonist, inhibits the proliferation of oral cancer cells. However, Marion et al. (31) stated that these agonists can also stimulate TLR7 expressing tumor cells so high TLR7 expression in the primary tumor confers poor clinical outcome and resistance to chemotherapy in lung cancer patients. This protumorigenic effect of TLR7 has been validated in murine models of lung carcinoma.

Multiple parameters could underlie this apparent discrepancy, such as the type of tumor, the level of TLR7 expression, the downstream function of TLR7 signaling in particular tumor cells, or chemotaxis of suppressive cells into the tumor. These major pathways of TLR7 stimulation could act either directly or indirectly on both immune and tumor cells, converging on cancer patient outcome and may affect the prognosis and the therapeutic approach of this tumor.

\section{CONCLUSION}

TLR7 expression was significantly correlated with the lymph node involvement and so can be used as a prognostic tool in OSCC cases.

\section{CONFLICT OF INTEREST}

The authors declare that they have no conflicts of interest.

\section{REFERENCES}

1. Ali J, Sabiha B, Jan HU, Haider SA, Khan AA, Ali SS. Genetic etiology of oral cancer. Oral Oncol. 2017;70:23-8.

2. Bray F, Ferlay J, Laversanne M, Brewster DH, Gombe Mbalawa C, Kohler B, et al. Cancer Incidence in Five Continents: Inclusion criteria, highlights from Volume $\mathrm{X}$ and the global status of cancer registration. Int J Cancer. 2015;137:2060-71.

3. Rivera C. Essentials of oral cancer. Int J Clin Exp Pathol. 2015;8:11884-94.

4. Choi S, Myers JN. Molecular pathogenesis of oral squamous cell carcinoma: implications for therapy. J Dent Res. 2008;87:14-32.

5. Torre LA, Bray F, Siegel RL, Ferlay J, Lortet-Tieulent J, Jemal A. Global cancer statistics, 2012. CA Cancer J Clin. 2015;65:87-108.

6. Ferlay J, Soerjomataram I, Dikshit R, Eser S, Mathers C, Rebelo $\mathrm{M}$, et al. Cancer incidence and mortality worldwide: sources, methods and major patterns in GLOBOCAN 2012. Int J Cancer. 2015;136:359-86

7. Luongo de Matos L, Manfro G, Vieira dos Santos R, Stabenow E, Sobroza de Mello E, Alves VAF, et al. Tumor Thickness As A Predictive Factor Of Lymph Node Metastasis And Disease Recurrence In T1n0 And T2n0 Squamous Cell Carcinoma Of The Oral Tongue. Oral Surgery, Oral Medicine, Oral Pathology, Oral Radiology. 2014.

8. Layland MK, Sessions DG, Lenox J. The influence of lymph node metastasis in the treatment of squamous cell carcinoma of the oral cavity, oropharynx, larynx, and hypopharynx: N0 versus N+. Laryngoscope. 2005;115(4):629-39.

9. Kanzler H, Barrat FJ, Hessel EM, Coffman RL. Therapeutic targeting of innate immunity with Toll-like receptor agonists and antagonists. Nat Med. 2007;13:552-9.

10. Pasare C, Medzhitov R. Toll-like receptors and acquired immunity. Semin Immunol. 2004; 16:23-6.

11. Yu L, Wang L, Chen S. Dual character of Toll-like receptor signaling: Pro-tumorigenic effects and anti-tumor functions. Biochimica et Biophysica Acta (BBA) - Reviews on Cancer. 2013; 1835:144-54.

12. Lee CC, Avalos AM, Ploegh HL. Accessory molecules for Toll-like receptors and their function. Nat Rev Immunol. 2012; 12:168-79.

13. Akira S. Toll receptor families: structure and function. Semin Immunol. 2004;16:1-2.

14. Takeda K, Kaisho T, Akira S. Toll-Like Receptors. Annual Review of Immunology. 2003;21:335-76.

15. Heil F, Hemmi H, Hochrein H, Ampenberger F, Kirschning C, Akira S, et al. Species-specific recognition of singlestranded RNA via toll-like receptor 7 and 8 . Science. 2004;303:1526-9.

16. Ahn MY, Kwon SM, Cheong HH, Park JH, Lee J, Min SK, et al. Toll-like receptor 7 agonist, imiquimod, inhibits oral squamous carcinoma cells through apoptosis and necrosis. J Oral Pathol Med. 2012;41:540-6.

17. Syrbu SI, Cohen MB. An enhanced antigen-retrieval protocol for immunohistochemical staining of formalinfixed, paraffin-embedded tissues. Methods Mol Biol. 2011;717:101-10.

18. Kirkpatrick LA, Feeney BC. A simple guide to IBM SPSS statistics for version 20.0. Student ed. Belmont, Calif.: Wadsworth, Cengage Learning; 2013.

19. Rosati G, Riccardi F, Tucci A. Use of tumor markers in the management of head and neck cancer. Int J Biol Markers 2000;15(2):179-83.

20. Guha N, Boffetta P, Wunsch F, V, Eluf NJ, Shangina O, Zaridze D, et al. Oral health and risk of squamous cell carcinoma of the head and neck and esophagus: results of two multicentric case-control studies. Am J Epidemiol 2007;166(10):1159-73.

21. Ahmed M.Kabel . Tumor markers of breast cancer: New prospectives. Journal of Oncological Sciences. 2017 April ; 3 (1):5-11.

22. Akira S, Takeda K. Toll-like receptor signalling. Nature Reviews Immunology. 2004;4:499.

23. Kaczanowska S, Joseph AM, Davila E. TLR agonists: our best frenemy in cancer immunotherapy. J Leukoc Biol. 2013;93(6):847-63.

24.Dajon M, Iribarren K, Cremer I. Toll-like receptor stimulation in cancer: A pro- and anti-tumor double-edged sword. Immunobiology. 2017;222(1):89-100.

25. Farzaneh Pakdel1, Firouz Pouralibaba1*, Sina Pakdel2, Reza Khorshidi Khiyavi3, Parisa Falsafi1, Hosein Eslami1 et al . Expression of toll-like receptors in squamous cell carcinoma of the tongue. Scholars Research Library. 2015; 7 (2):20-24

26. Shiva Solahay Kahnamouii, Firoz Pouralibaba, Farzaneh Pakdel , Mahni Rahkar Farshi, Farrokh Farhady , Homayoon Sadegi Bazargani et al. Expression of The Toll Like Receptor (TLR) 2, TLR4, TLR7 and TLR8 in Squamous Cell Carcinoma of the Human Tongue. Oral Surgery, Oral Medicine, Oral Pathology and Oral Radiology. 2015;119(3):106. 
27. Ilyar Sheyhidin, Gulnaz Nabi, Ayshamgul Hasim, Rui-Ping Zhang, Julaiti Ainiwaer, Hong Ma . Overexpression of TLR3, TLR4, TLR7 and TLR9 in esophageal squamous cell carcinoma. World J Gastroenterol. 2011 August ; 17(32): 3745-3751.

28. Julien Cherfils-Vicini, Sophia Platonova, Mélanie Gillard, Ludivine Laurans, Pierre Validire, Rafaele Caliandro. Triggering of TLR7 and TLR8 expressed by human lung cancer cells induces cell survival and chemoresistance. J Clin Invest. 2010;120(4):1285-1297.

29. Saradiya Chatterjee, Lucile Crozet, Diane Damotte, Kristina Iribarren,Catherine Schramm, Marco Alifano . TLR7 Promotes Tumor Progression, Chemotherapy Resistance, and Poor Clinical Outcomes in Non-Small Cell Lung Cancer. Cancer Research. 2014 septemper ;74(18): 5008-18.
30. Mee-Young Ahn, Seong-Min Kwon, Hak Hyun Cheong, Jong-Hwan Park Ren WH, Toll-like receptor 7 agonist, Imiquimod, inhibits oral squamous carcinoma cells through apoptosis and necrosis. Journal of Oral Pathology and Medicine. May 2012; 41(7):540-6.

31. Marion Dajon, Kristina Iribarren, and Isabelle Cremer. Dual roles of TLR7 in the lung cancer microenvironment. OncoImmunology . March 2015 ;4 (3) :1615. 